\title{
Gigahertz-peaked spectra pulsars
}

\author{
Karolina Rożko ${ }^{1}$, Wojciech Lewandowski ${ }^{1}$, Jarosław Kijak ${ }^{1}$ \\ and Rahul Basu ${ }^{1,2}$ \\ ${ }^{1}$ Janusz Gil Institute of Astronomy, University of Zielona Góra, \\ ul. Szafrana 2, 65-516 Zielona Góra, Poland \\ email: krozko@gmail.com \\ ${ }^{2}$ Inter-University Centre for Astronomy and Astrophysics \\ Pune, 411007, India \\ email: rahulbasu. astro@gmail.com
}

\begin{abstract}
In this short overview we summarize our knowledge of twenty five pulsars showing GPS characteristics. Especially, we will focus on two objects. The first is PSR B1800-21 - a Vela-like GPS pulsar with a variable spectrum. The second is PSR J1740+1000 - a pulsar that shows high frequency turnover based on our most recent observations using the Giant Meterwave Radio Telescope and the Green Bank Telescope.
\end{abstract}

Keywords. pulsars: general, ISM: general, pulsars: individual (J1740+1000, B1800-21)

\section{Introduction}

The pulsar spectra in most cases can be described by a simple power-law function, with the population average spectral index close to -1.8 (Maron et al. 2000), but some pulsars show a different behaviour. The first direct evidence of the turnover in pulsar spectrum at high frequency were published by Kijak, Gupta \& Krzeszowski (2007). Later, they were named gigahertz-peaked spectra (Kijak et al. 2011b).

Presently there are 25 confirmed GPS pulsars known out of which 18 are associated with either supernova remnant, a pulsar wind nebula, an HII region or an unidentified HESS source (the full list of the GPS pulsars is available in the article by Kijak et al., in the same proceedings). This fact allowed us to form a hypothesis that the apparent low frequency flux density deficit is caused by some external mechanism (see Kijak et al. 2011b). This hypothesis was further strengthened by the observations of the binary system comprising of PSR B1259-63 and Be star (see Kijak et al. 2011a and references therein). In this system the pulsar spectra were observed at different epochs with the pulsar at different locations with respect to the Be star. When the pulsar was far away from the star its spectrum looked like a typical "power-law", while closer to the periastron passage it showed a turnover. In this case the absorption can happen either in the stellar wind or the disc around the Be star. The only type of absorption that could work in such environment is the free-free thermal absorption on the electrons of the wind or disc.

The thermal absorption depends on the electron temperature, the observed frequency, the electron density and the physical thickness of the absorber (see Rybicki \& Lightman 1979 and Wilson, Rohlfs \& Hüttemeister 2009 for details). Hence the strongest absorption will be caused by the dense, cold but ionized regions. The free-free thermal absorption was proposed previously by Sieber (1973) to explain the low frequency turnover around $100 \mathrm{MHz}$ seen in certain pulsars. We have adapted this model in our studies e.g. a more detailed study of the spectral shape using the model has been conducted in 17 pulsars by Kijak et al. (2017). In this article we will focus on the two sources: J1740+1000 (a young 

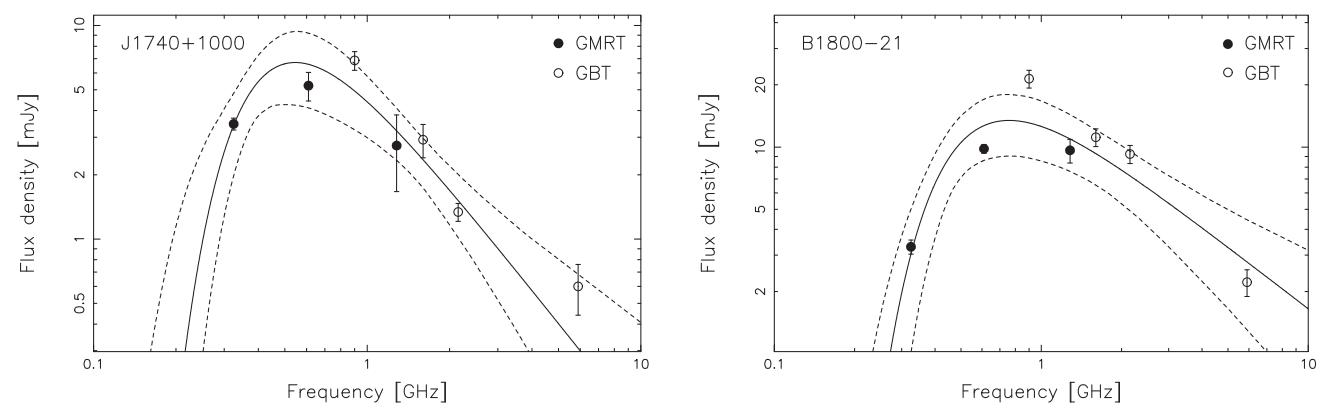

Figure 1. The pulsars spectra with fitted free-free thermal absorption model. The dashed lines correspond to $1 \sigma$ envelope. The fitted parameters are presented in the Table 1.

pulsar far from the galactic plane) and B1800-21 (a Vela-like pulsar near W30 complex). The low frequency spectra of these two sources have been studied in the past (Kijak et al. 2011b, Dembska et al. 2014, Bilous et al. 2016, Basu et al. 2016). In case of J1740+1000 there was confusion if the spectrum actually showed turnover. While the spectral turnover of B1800-21 showed variations at multiple observing epochs. In this work we present results of a multifrequency, contemporaneous observations that characterise the spectral nature of the two sources.

\section{Observations and results}

The pulsars J1740+1000 and B1800-21 were observed using two radiotelescopes: the Giant Meterwave Radio Telescope (GMRT) located near Pune in India and the Green Bank Telescope (GBT) located in USA (the details of these observations can be found in Rożko et al. 2017). Using GMRT we observed both pulsars at three frequencies: 325 $\mathrm{MHz}, 610 \mathrm{MHz}$ and $1200 \mathrm{MHz}$. For each frequency we conducted three observational sessions separated by at least one week to account for the possible influence of interstellar scintillations on our flux density measurements. Observations were held between 1 August 2016 and 3 September 2016. The GBT observations covered four frequencies: $900 \mathrm{MHz}$, $1600 \mathrm{MHz}, 2150 \mathrm{MHz}$ and $5900 \mathrm{MHz}$. Observations were conducted between 12 December 2016 and 17 January 2017.

We modelled the spectra using the free-free thermal absorption model. The fitted parameters were: A - the scaling parameter of the intrinsic pulsar flux, B - the parameter that describes the frequency-independent part of the optical depth $B=0.08235 \times$ $T_{\mathrm{e}}^{-1.35} \mathrm{EM}$ and $\alpha$ - the spectral index of the intrinsic pulsar flux. The results of our fits are presented in Fig. 1 and in the left side of Table 1. Following Basu et al. (2016) and Kijak et al. (2017) we used the pulsars' dispersion measure (DM) to get some constraints on the electron density and temperature of the absorber. We considered three cases: a dense supernova remnant filament (with size equal $0.1 \mathrm{pc}$ ), the pulsar wind nebula (with the size equal to $1.0 \mathrm{pc}$ ) and a cold H II region (with size equal $10.0 \mathrm{pc}$ ) as possible source of absorbing medium. We estimated constraints on the different physical parameters of the three potential absorbing medium as showed in the right side of Table 1.

\section{Discussion and conclusions}

PSR J1740+1000 is a young pulsar located at a relatively large distance from the galactic plane and thus has a very low value of $\mathrm{DM}=24 \mathrm{pc} \mathrm{cm}$ (McLaughlin et al. 2000). It is also associated with an X-ray PWN with very extended tail (see Kargaltsev 
et al. 2008 and Kargaltsev \& Pavlov 2010). Shortly after the discovery McLaughlin et al. (2002) reported that the flux density measurements were affected by very strong diffractive scintillations in the L-Band frequency range. That problem made the interpretation of its spectrum very difficult in the past. The ambiguity of the interpretation of that pulsar spectrum was further increased by Bilous et al. (2016), who added the $150 \mathrm{MHz}$ flux density measurement from LOFAR observations, which allowed them to propose a simple power-law interpretation of J1740+1000 spectrum. However, they made a single 30-minute observation and the profile upon which the measurement was based had very low signal-to-noise ratio. For that reasons we found the reported flux density value as doubtful.

Our latest observational results support the GPS interpretation for this pulsar spectrum (see Fig. 1). The most probable absorber is a partially ionized small molecular cloud in front of the pulsar which is supported by the the electron density value derived from DM for the absorber thickness of $0.1 \mathrm{pc}$ (i.e. $119 \mathrm{~cm}^{-3}$ ). The derived electron density value is consistent with the electron density in front of the shock in the case of some of the bow-shock PWNe that were estimated from optical observations of atomic emission lines to be of the order of $50-100 \mathrm{~cm}^{3}$ (Hester \& Kulkarni 1989 and Li et al. 2005).

Looking at the physical constraints presented in the right side of Table 1 we can rule out an H II region as a possible absorber, because the estimated temperature is unphysical for that type of structure. On similar grounds absorption caused by the electrons located inside the bow-shock PWN is doubtful, but not impossible. In the case of PSR J1740+1000 we are probably looking at the PWN from its side where the thermal absorption, if present, should be relatively weak (see Lewandowski et al. 2015 for explanation).

A spectral model based exclusively on our latest measurements supports the GPS interpretation. We want to point out, that almost all previous measurements from LBand were sparsly observed with a large intervals between them. In our studies we have used multiple, equally spaced observations and estimated their average which should serve as a better measurements for the flux at L-Band. However, if we use all of the available flux density measurements to model the spectrum the results are not so clear. Taking into account $150 \mathrm{MHz}$ LOFAR measurements still did not allow us to exclude a simple power-law interpretation. Therefore, only an independent flux density measurement at frequencies around or below $200 \mathrm{MHz}$, using much longer integration time, or possibly a different method (such as interferometric imaging) can be decisive in this case.

PSR B1800-21 is a Vela-like pulsar located in a very peculiar environment called W30 complex, which is a supernova remnant with a large number of $\mathrm{H}$ II regions around it (Kassim \& Weiler 1990). Basu et al. (2016) conducted a detailed study of the spectral changes of this object. They showed that turnover in the spectrum of B1800-21 shifted from lower to higher frequency over a time scale of a few years. They explained such

Table 1. The fitted parameters for the spectra of PSR J1740+1000 and PSR B1800-21 using the thermal absorption model and the constraints on the physical parameters of the absorbing medium.

\begin{tabular}{lcccc|ccc}
\hline PSR name & Amp & $\mathrm{B}$ & $\alpha$ & $\nu_{\mathrm{p}}[\mathrm{GHz}]$ & Size $[\mathrm{pc}]$ & $\mathrm{n}_{\mathrm{e}}\left[\mathrm{cm}^{-3}\right]$ & $\mathrm{T}_{\mathrm{e}}[\mathrm{K}]$ \\
\hline $\mathrm{J} 1740+1000$ & $0.132_{-0.094}^{+0.275}$ & $0.22_{-0.12}^{+0.11}$ & $-1.61_{-0.63}^{+0.66}$ & 0.55 & 0.1 & $119.48 \pm 0.13$ & $106_{-45}^{+42}$ \\
& & & & & 1.0 & $11.948 \pm 0.013$ & $19.3_{-8.6}^{+7.6}$ \\
& & & & & 10.0 & $1.1948 \pm 0.0013$ & $3.5_{-1.5}^{+1.4}$ \\
\hline B1800-21 & $1.65_{-1.05}^{+1.52}$ & $0.26_{-0.10}^{+0.15}$ & $-1.00_{-0.49}^{+0.39}$ & 0.76 & 0.1 & $1169.95 \pm 0.25$ & $2680_{-770}^{+1100}$ \\
& & & & & 1.0 & $116.995 \pm 0.025$ & $488_{-199}^{+199}$ \\
& & & & & 10.0 & $11.6995 \pm 0.0025$ & $89_{-25}^{+36}$ \\
\hline
\end{tabular}


transition by assuming an additional absorption that happened during that time. The most probable absorber in this case is a small and dense supernova remnant filament that crossed our line of sight. Our latest observations confirm that the turnover frequency came back to the original value (i.e prior to the 2012 change). This would suggest that the spectral change observed between 2012 and 2014 was indeed a rare event, rather than a continuous variation, which further supports the filament crossing the line of sight interpretation.

Summary. Today we know 25 GPS pulsars (including B1259-63, J1740+1000 and 3 radiomagnetars) and there is considerable evidence that an external mechanism is responsible for the spectral turnovers. The most compelling possibility is the thermal free-free absorption taking place in pulsar environments (see Lewandowski et al. 2015, Rajwade, Lorimer \& Anderson 2016, Basu et al. 2016 and Kijak et al. 2017).

\section{Acknowledgments}

We thank the staff of the GMRT who have made these observations possible. The GMRT is run by the National Centre for Radio Astrophysics of the Tata Institute of Fundamental Research. This research was partially supported by the grant DEC2013/09/B/ST9/02177 of the Polish National Science Centre.

\section{References}

Basu, R., Rożko, K., Lewandowski, W., Kijak, J., \& Dembska, M. 2016, MNRAS, 458, 2509

Bilous, A. V., Kondratiev, V. I., Kramer, M. et al. 2016, A\&AA, 591, A134

Dembska, M., Kijak, J., Lewandowski, W., Jessner, A., Bhattacharyya, B., \& Gupta, Y. 2014, $M N R A S, 445,3105$

Hester, J. J. \& Kulkarni, S. R. 1989, ApJ, 340, 362

Kargaltsev, O., Misanovic, Z., Pavlov, G. G., Wong, J. A., \& Garmire, G. P. 2008, ApJ, 684, $542-557$

Kargaltsev, O. \& Pavlov, G. G. 2010, AIP-CP, 1248, 25-28

Kassim, N. E. \& Weiler, K. W. 1990, ApJ, 360, 184

Kijak, J., Gupta, Y., \& Krzeszowski, K. 2007, A\&A, 462, 699-702

Kijak, J., Dembska, M., Lewandowski, W., Melikidze, G., \& Sendyk, M. 2011a, MNRAS (Letters), 418, L114-L118

Kijak, J., Lewandowski, W., Maron, O., Gupta, Y., \& Jessner, A. 2011b, AE\&A, 531, A16

Kijak, J., Basu, R., Lewandowski, W., Rożko, K., \& Dembska M. 2017, ApJ, 840, 108

Lewandowski, W., Rożko, K., Kijak, J., \& Melikidze, G. I. 2015, ApJ, 808, 18

Li, X. H., Lu, F. J., \& Li, T. P. 2005, ApJ, 628, 931

Maron, O., Kijak, J., Kramer, M., \& Wielebinski, R. 2000, A\&A, 147, 195

McLaughlin, M. A., Cordes, J. M., \& Arzoumanian, Z. 2000, in: M. Kramer, N. Wex \& R. Wielebinski (eds.), Pulsar Astronomy - 2000 and Beyond, IAU Colloquium 177 (San Francisco: Astronomical Society of the Pacific), p. 41

McLaughlin, M. A., Arzoumanian, Z., Cordes, J. M., Backer, D. C., Lommen, A. N., Lorimer, D. R., \& Zepka, A. F. 2002, ApJ, 564, 333

Rajwade, K., Lorimer, D. R., \& Anderson, L. D. 2016, MNRAS, 455, 493

Rożko, K., Rajwade, K., Lewandowski, W., Basu, R., Kijak, J., \& Lorimer, D. R. 2017, in preparation

Rybicki, G. B. \& Lightman, A. P. 1979, Radiative Processes in astrophysics (New York, Chichester, Brisbane, Toronto, Singapore: John Wiley \& Sons, Inc.)

Sieber, W. 1973, A\& A, 28, 237

Wilson, T. L. \& Rohlfs, K., Hüttemeister S. 2009, Tools of Radio Astronomy (Berlin: Springer) 